\title{
Gestational Diabetes and Cold Stress Trigger Protein Oxidation in Discrete Brain Regions
}

\author{
Rizwan Sharief and Mahaboob Basha $\mathrm{P}^{*}$ \\ Department of Zoology, Bangalore University, Bangalore- 560 056, India
}

\begin{abstract}
Gestational diabetes is unique because of the diversity of problems that can affect the embryo/fetus beginning with conception. Streptozotocin (STZ), a diabetogenic agent when administered to pregnant rats in high dose, induces diabetes by destructing pancreatic $\beta$-islet cells resultantly in the intrauterine life of developing fetuses limits their adaptation with depleted insulin secretion. Similarly, environmental stressors like cold-stress result in fetal hypoinsulinemia with a reduction in the number of insulin receptors on target cells. In a given situation, if both stressors are prevailing, the resultant free radical production in prenatal life may bring severe oxidative stress on the molecular integrity of proteins that might progress to weaning and adulthood. In this study, the oxidative indices measured in STZ induced gestational rats upon exposure to cold stress $\left(15^{\circ} \mathrm{C} \& 20^{\circ} \mathrm{C}\right)$ indicate significant changes in discrete brain regions. Cold-stress found to exacerbate the free radical production in diabetic subjects and impose a higher rate of protein oxidation confirming synergetic effects. The findings for the first time confirm that the oxidative changes that occurred due to prenatal stress remain into weaning and adulthood, specifically in the functional areas like the cerebral cortex and hippocampus, which in turn may bring impairments/deficits in memory and cognitive processes.
\end{abstract}

Keyword:- Gestational diabetes, Cold stress, Developing brain, Free-radicals, Protein oxidation, Synergistic effects.

\section{INTRODUCTION}

Gestational diabetes (GD) is a glucose intolerance state wherein consistent hyperglycemia is observed during pregnancy [1]. It is usually distinguished during $2^{\text {nd }}$ or early $3^{\text {rd }}$ trimester, arising from insufficiency of insulin secretion or lack of response to insulin [2]. As per an estimate, about 21.3 million live births (16.2\%) suffering from some sort of hyperglycemia during pregnancy [3]. Assessment of human cohort studies and research using rodent models have demonstrated that tenacious hyperglycemia during pregnancy and critical stages, cause cognitive deficits in offspring [4-6]. Technically, diabetes modifies homeostasis and cause oxidative burden in vulnerable tissues by enhancing reactive oxygen species [7-8]. Moreover, prenatal development is sensitive to environmental influences. More directly, Hanssen et al. [9] showed the impacts of cold acclimation on insulin sensitivity in type-2 diabetes subjects, an impact that was related to markedly increased striated muscle GLUT-4 translocation. It's well established that severe cold exposure causes marked whole- body cooling and thus impairs the function of organs [10]. Membrane injury following lipid peroxidation disrupts tissue integrity, therefore to convert ROS into less reactive species, developing tissues might have evolved efficient stress responses by activating protein control systems. In the intrauterine environment, chronic hyperglycemia swayed some metabolic changes within the offspring, including increased weight, early postnatal period, cognitive deficits, which might be aggravated by cold stress. Ongoing multiple stressors posing their effects on intrauterine life and placenta being the target of increased sympathetic tone during gestation, there is a possibility of functional vulnerabilities that may contribute to the pathogenesis in post-natal life. However, the mechanistic reasons involving interaction of several factors, such as the timing of exposure, duration and ultimate outcome in offspring behaviour is not explored fully. Besides, in utero studies on stress-induced changes in response to cold exposure in diabetic subjects are obscure, hence this study was undertaken to unravel the changes occurring in discrete regions of brain tissue of offspring born as a function of cold stress to DM subjects.

\section{MATERIAL AND METHODS}

\section{A. Chemicals}

Streptozotocin (STZ), Serum albumin (BSA), Guanidine hydrochloride were obtained from Sigma Aldrich, USA. 5,5'-Dithio-bis (2-nitrobenzoic-acid) (DTNB) purchased from Merck India Ltd. All other chemicals were of analytical grade and were purchased from SD fine chemicals Ltd, India and SISCO research laboratories (SRL), India.

\section{B. Animals}

Laboratory acclimated healthy albino rats (200-250g) procured from Sri Raghavendra Enterprises, Bangalore (841/PO/Bt/S/04/CPCSEA/2017-22) were inducted to the diabetic state by a single-dose intraperitoneal injection of STZ (50 mg/kg bw in $0.1 \mathrm{~mol} / \mathrm{l}$ citrate buffer $\mathrm{pH} 4.5)$. Three days-post STZ administration, blood samples were drawn from the tail vein and glucose levels were tested (AccuCheck Active Glucometer) and rats confirmed diabetic when their fasting blood glucose levels were more than 200 $\mathrm{mg} / \mathrm{dL}$ were selected for the experimentation. These rats along with the control group were allowed to breed separately ( 3 females: 1male ratio) and were examined for the vaginal plugs, wherein females confirmed positive for pregnancy were recorded as day-1 of gestation accordingly diabetic subjects were called GDM (Gestational diabetic mellitus) and control group as non-diabetic. 


\section{EXPERIMENTAL DESIGN}

All of the experimental procedures complied with National Institution of Nutrition, Hyderabad (Guidelines for the Care and Use of Laboratory Animals) and were approved by the Bioethics Committee of the Faculty of Zoology at Bangalore University, Bangalore (Protocols number: DOZ/BUB/2018-19 and 402/CPSCSEA 2009-12 $\&$ revival thereon). Every effort was made to minimize the quantity of animals used and their suffering. The gestational rats on the day- 1 were made into groups viz., control (Group-I); cold stress (Group-II \& III); and diabetic (Group-IV). The control rats were maintained at room temperature during their entire pregnancy and cold stress (3hr/day) was applied to Group-II \& III animals by subjecting in a cold stress chamber (Colton, India) at $15^{\circ} \mathrm{C}$ $\& 20{ }^{\circ} \mathrm{C}$ respectively during entire pregnancy. Likewise, GDM rats were further grouped as Group-V \& VI to induce cold stress at $15^{\circ} \mathrm{C} \& 20^{\circ} \mathrm{C}$ respectively. Post- parturition, care was taken to maintain litters along the dam for a month and used for assessment.

\section{A. Biochemical Analysis}

One-month-old male pups $(n=6)$ representing from each group were euthanized by spinal dislocation under 1 $\%$ pentobarbital sodium $(0.4 \mathrm{~mL} / 100 \mathrm{~g} \mathrm{bw})$ anaesthesia and discrete brain regions viz., cerebral cortex (CC), cerebellum (CB), hippocampus $(\mathrm{H})$, medulla oblongata $(\mathrm{M})$ and spinal cord (SC) were isolated and homogenized in requisite buffers. Upon centrifugation, the aliquots were used to determine the following biochemical parameters connected to oxidative stress and protein oxidation.

\section{Lipid peroxidation}

The extent of lipid peroxidation was measured by 2thiobarbituric acid-reactive substances (TBARS) as described by Niehaus and Samuelsson [11]. with slight modification. The assay mixture containing $1.0 \mathrm{~mL}$ of tissue extract and $2 \mathrm{~mL}$ of TCA-TBA-HCl mixture $(15.00$ \% TCA: $0.37 \%$ TBA: $0.25 \mathrm{~N} \mathrm{HCl}=1: 1: 1)$, was placed in boiling water for $15 \mathrm{~min}$. Further, the reaction mixture was cooled and centrifuged at $1000 \mathrm{rpm}$ for $10 \mathrm{~min}$. The absorbance of the clear supernatant was read at $535 \mathrm{~nm}$ using UV/Vis spectrophotometer. The amount of formation of MDA content was measured and expressed as ' $\mu$ moles/g'.

\section{$>$ Protein carbonyl levels}

The protein carbonyl (PCO) groups were measured spectrophotometrically by adopting the method given by Reznick and Packer [12]. In the estimation, the carbonyl compound reacts with 2,4-dinitrophenylhydrazine forming 2,4-dinitrophenylhydrazone whose intensity was detected at $375 \mathrm{~nm}$ spectrophotometrically. Tissue homogenates (1.0 $\%$, w/v) were prepared in $20 \mathrm{mM}$ sodium phosphate buffer (pH 6.5), and aliquots were separated upon centrifugation. About $200 \mu \mathrm{L}$ of aliquot was extracted with $10 \%$ TCA $(500 \mu \mathrm{L})$ centrifuged further at $5000 \mathrm{rpm}$ for $10 \mathrm{~min}$. The precipitate obtained was treated with $500 \mu \mathrm{L}$ of DNPH $(0.2$ $\%$, w/v in $2 \mathrm{~N} \mathrm{HCl}$ ) and incubated at room temperature for $1 \mathrm{hr}$ by vortexing at 5 -min interval. The proteins than precipitated by adding $55 \mu \mathrm{L}$ of $100 \%$ TCA. The pellet was centrifuged and washed three times with ethanol: ethyl acetate mixture $(500 \mu \mathrm{L})$ and finally dissolved in $600 \mu \mathrm{L}$ of $6 \mathrm{M}$ guanidine hydrochloride and left for $10 \mathrm{~min}$ at $37{ }^{\circ} \mathrm{C}$ by continuous vortexing. The carbonyl content was measured at $375 \mathrm{~nm}$ against the blank. The 2,4dinitrophenylhydrazone protein adducts were calculated using the $\mathrm{mM}$ absorptivity of $22.0 \mathrm{mM}-1 \mathrm{~cm}-1$ for aliphatic hydrazones and results expressed as 'nmoles / mg protein'.

\section{Total thiol groups}

Protein thiol content(s) was determined by adopting the method given by Lou et al. [13]. Thiol groups which are linked to proteins by disulphide bonds, gets released during reduction process and the acid-soluble thiols are readily separated by precipitating the protein with PCA, then sulfhydryl's present in the protein-free extract of the sample reacts non-enzymatically with Ellman's reagent (DTNB) to yield GSSG and TNB (2-nitro-5-thiobenzoic acid). For the assay, tissue homogenates $(10 \mathrm{mg})$ were suspended in $1 \mathrm{~mL}$ of $\mathrm{HClO} 4(0.3 \mathrm{M})$ containing $5 \mathrm{mM}$ EDTA and $0.06 \mathrm{~g} / \mathrm{L}$ bipyridine, and centrifuged for $15 \mathrm{~min}$ at $8000 \mathrm{rpm}$. The pellet was washed with the same solution and then with $1 \mathrm{~mL}$ of ethanol-ethyl acetate $(1: 1, \mathrm{v} / \mathrm{v})$. The final pellet dried under nitrogen and suspended in $0.2 \mathrm{~mL}$ of $6 \mathrm{M}$ guanidine hydrochloride and $0.1 \mathrm{~mL}$ of $0.15 \mathrm{M}$ $\mathrm{KH} 2 \mathrm{PO} 4$ at $\mathrm{pH} 7.4$. The aliquots of the protein solution (75 $\mu \mathrm{L}$ ) were mixed with $0.925 \mathrm{~mL}$ of $50 \mathrm{~m} M \mathrm{KH} 2 \mathrm{PO} 4, \mathrm{pH} 7.4$ containing $5 \mathrm{mM}$, EDTA and $1 \mathrm{~mL}$ of $2 \mathrm{~m} M$ DTNB. After 30 min of incubation, the absorbance was measured at 412 $\mathrm{nm}$ at room temperature. Reduced glutathione was used as a standard. The concentration of $\mathrm{P}-\mathrm{SH}$ groups in the samples was calculated using a molar absorption coefficient of $136 \mathrm{mM}-1 \mathrm{~cm}-1$ and results expressed as ' $\mu$ moles/mg protein'.

\section{B. Data Interpretation and Statistical Analysis}

Statistical analysis was carried out by SPSS software 20.0 software. One-way Analysis of Variance (ANOVA) with post hoc test was performed for the inter-group comparisons using Duncan's Multiple Range Test using SPSS 20.0 at probability (P) value 0.05 level of significance. Graphs were plotted using 'Origin Pro' software 9.0

\section{RESULT}

\section{$>$ Lipid peroxidation}

The changes occurred in the levels of MDA in discrete brain regions of experimental and control group animals were shown in figure- 1 and it is clear from data that the levels of MDA significantly $(\mathrm{P}<0.05)$ increased in discrete brain regions of experimental rats studied upon exposure to cold stress as well as in diabetic group, while exacerbated effects were witnessed in dual stressor groups (diabetes \& cold stress at $15^{\circ} \mathrm{C}$ and $20^{\circ} \mathrm{C}$ ). It is also evident from the results that the cerebral cortex and hippocampus are found to be highly vulnerable to oxidative damage than other regions studied such as the cerebellum, medulla, and spinal cord. 


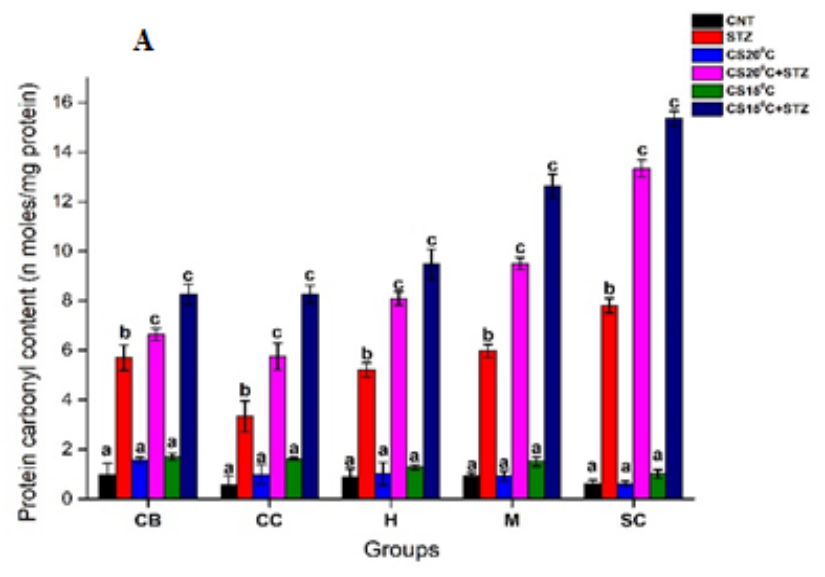

\section{$>$ Protein oxidation}

The data on variations that occurred in protein carbonyls and total thiol content in discrete brain regions upon exposure to cold stress in diabetic rats are shown in figure $(2 \& 3)$. A significant $(\mathrm{P}<0.05)$ increment in protein carbonyl content was evident in discrete brain regions of rats in diabetic and cold-stressed (both $15^{\circ} \mathrm{C}$ and $20{ }^{\circ} \mathrm{C}$ ) subjects indicating plausible damage of proteins that alter many physiological functions. Likewise, exposures at $20{ }^{\circ} \mathrm{C}$ were found to cause higher carbonyl production compared to $15^{\circ} \mathrm{C}$. It is also clear from the results that cold stress exposure displayed exacerbated effect in augmenting higher protein carbonyl production in individual exposures of cold stress as well STZ induced hyperglycemic state. A significant $(\mathrm{P}<0.05)$ decrease in protein thiol content was observed in discrete brain regions of diabetic and coldstressed rats (both $15{ }^{\circ} \mathrm{C}$ and $20{ }^{\circ} \mathrm{C}$ ), while exacerbated effects were witnessed in dual stressor groups (diabetes \& cold stress at $15^{\circ} \mathrm{C}$ and $20{ }^{\circ} \mathrm{C}$ ). Among discrete regions studied medulla and spinal cord showed higher vulnerable to oxidative damage than other regions such as the cortex, cerebellum and hippocampus.

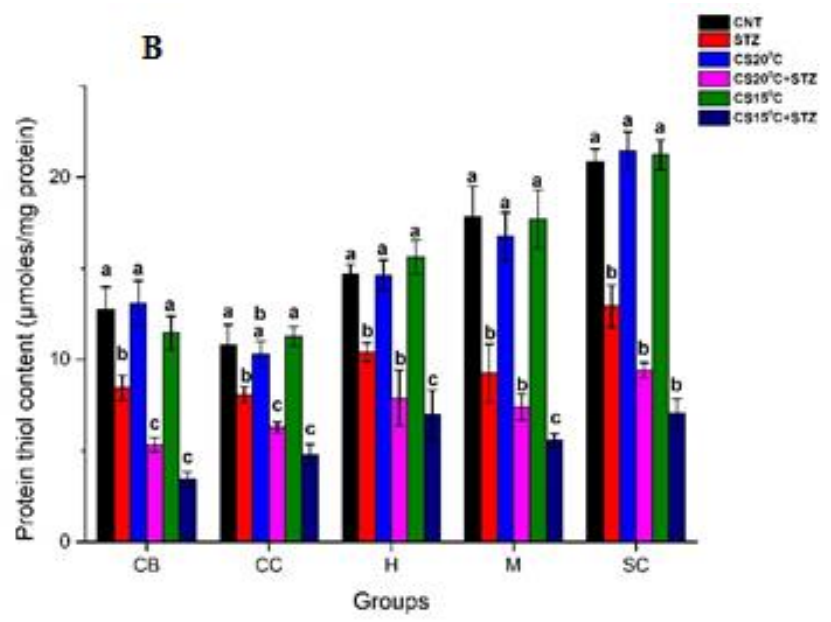

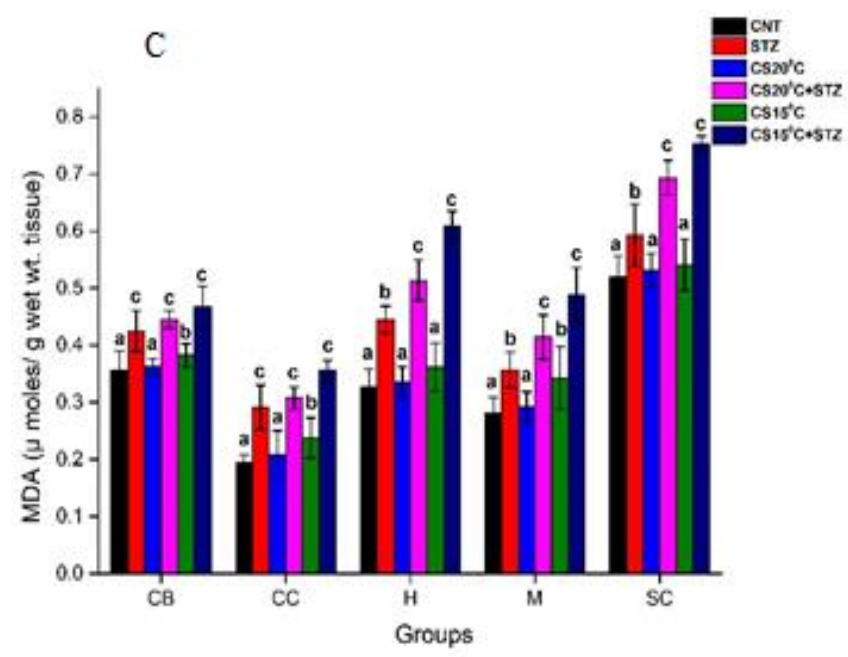

Figures:- A, B \& C represents the level of Protein Carbonyl, Protein thiol and MDA contents respectively in discrete rat brain regions of gestational diabetic rats as a function of exposure to cold stress $\left(15\right.$ and $\left.20{ }^{\circ} \mathrm{C}\right)$. Values are represented in mean $\pm \operatorname{SEM}(n=6)$. alphabets ' $a$ ', ' $b$ ' and 'c' represents significantly different among experimental groups as determined by DMRT at significance $\mathrm{P}<0.05$. Abb: CB-Cerebellum, CC-Cerebral cortex, H-Hippocampus, M-Medulla, SC- Spinal cord.

\section{DISCUSSION}

The chronic hyperglycemia and Insulin resistance of diabetes type-2 leads to damage or dysfunction of functional organs. Growing evidence suggests that freeradicals have a vital effect on the pathophysiology of diabetes progression and complications as hyperglycemia is associated with a gradual rise in oxidative stress [14]. The subjects, who develop GDM experience a shortfall in pancreatic $\beta$ cell response, leading to inadequate insulin supply resulting in the state of hyperglycemia and when not taken care during pregnancy, it impairs the intrauterine environment affecting the normal fetal development resulting in long-term effects on the structure and function of an organ system(s). Relevant studies also indicated cold exposure enhanced oxidative stress by increasing the prooxidants while depleting the antioxidant capacities. For our knowledge, this is the first study, wherein the influence of cold stress on gestational diabetes is being examined; given the stress conditions, free-radicals bring oxidative changes on macromolecular integrity, thereby we have measured and compared concentrations of LPO product the MDA, total thiols, and protein carbonyls from discrete brain regions of gestational diabetic rats as a function of hypothermia (cold stress). 


\section{$>$ Lipid peroxidation}

The brain is an organ that is highly sensitive to oxidative damage because of its high metabolic activity, high lipid content, and the limited activity of antioxidant defense mechanisms [15]. Gestation is known to enhance the state of oxidative stress as a result of high metabolic activity and overproduction of ROS causing massive cellular impairment. To assess if there is an increase in oxidants that cause oxidative damage in lipids, we evaluated lipid peroxidation and observed a consistent increase in MDA levels in all experimental rats, confirming oxidative stress in brain functional tissues. Using MDA as a marker, Huerta-Cervantes et al., [16] reported gestational diabetes triggered oxidative stress in the hippocampus and cerebral cortex and cognitive behavior modifications in rat offspring. Their results suggest that intrauterine milieu with chronic hyperglycemia causes oxidative stress and alter cognitive behavior in an age- and sex-dependent manner and any reduction in ROS burden contributes to decreasing oxidative damage in developing embryos of rats with gestational diabetes [16]. In this study, the MDA levels increased significantly in discrete brain regions in both diabetic and cold-stressed offspring, thereby the results of this study corroborate with the findings of recent studies of Huerta-Cervantes et al. [16]. Further, exacerbated effects witnessed as a function of cold stress in diabetic rats, infer synergetic actions due to bioaccumulation of LPO metabolites and their interference with the developing neuronal tissues because of poor antioxidant defense; resultantly cold stress intensifies the oxidative burden in diabetic rats causing severity of effects in developing brain because optimal brain function is dependent on cellular redox homeostasis and these changes initiate tissue damage. These results also suggest that the cerebral cortex and hippocampus regions are highly vulnerable to oxidative damage and therefore liable to present an altered function compared to other regions like the cerebellum, medulla, and spinal cord. These findings indicate region-specific alterations in the antioxidant system in discrete functional tissues of the brain, which remained into adulthood that may lead to behavioral/cognitive alterations.

\section{$>$ Protein oxidation}

The oxidative modification of proteins is implicated in the etiology or progression of a panoply of disorders and diseases. Oxidative damage to proteins by ROS results in cleavage of the polypeptide chain and their cross-linking that leads to the modification of amino acid side chains in the protein structure. Cleavage of the peptide by ROS results in the formation of carbonyl groups [17] thereby oxidative damage induced changes in proteins is considered to be a biomarker of oxidative stress [18]. The covalent modification of the protein happens either directly by ROS or indirectly by reacting with the secondary by-products of oxidative stress. Thiols are physiological free radical scavengers, serves as an antioxidant(s) by several mechanisms [19]. They are important components of the total redox buffer system [20] and are very protective against oxidative stress [21], [22], [23]. Upon exposure to cold stress, the dam (mother) and neonates are likely to have excessive free radical production as a result of placental oxidative stress resultant significant $(\mathrm{P}<0.05)$ increments in protein carbonyl content observed in discrete brain regions in rats of diabetic and cold-stressed (both 15 ${ }^{\circ} \mathrm{C}$ and $20{ }^{\circ} \mathrm{C}$ ) subjects, indicating the plausible damage of proteins that alter many physiological functions. Likewise, exposures at $20{ }^{\circ} \mathrm{C}$ were found to cause higher carbonyl production compared to $15{ }^{\circ} \mathrm{C}$. It is also clear from the results that cold stress exposure (both $15^{\circ} \mathrm{C}$ and $20{ }^{\circ} \mathrm{C}$ ) exhibited exacerbated effect in augmenting higher protein carbonyl production in individual exposures of cold stress as well STZ induced hyperglycemic state. Besides, oxidation of proteins has shown to be perpetuated to another compound viz., the thiol comprising cysteine, which is liable to oxidation. Resultantly higher vulnerability was evident by exhibiting a decrease in protein thiol content in both diabetic and cold-stressed rats, while dual stressors (diabetes \& cold stress at $15^{\circ} \mathrm{C}$ and 20 ${ }^{\circ} \mathrm{C}$ ) exhibited exacerbated effect in suppressing thiol content in discrete brain regions studied. These changes in carbonyl and thiol contents may lead to accelerated protein degradation and increased catabolism or suppression of protein synthesis. To strengthen our inferences, studies of Petrone et al. [24] have suggested individuals with diabetes be potentially more susceptible to the consequences of cold stress. Most research in this area has advocated possible soothing effects of cold exposure for individuals with type2 diabetes. [2], [25], [26], [27]. Hanssen et al. [9] reported that only 10 days of cold acclimation $\left(14-15^{\circ} \mathrm{C}\right.$ ) in individuals with type- 2 diabetes induction brought about $43 \%$ increase in insulin sensitivity, which was explained by a $60 \%$ increase in GLUT-4 translocation (i.e., the membrane channel that allows glucose to enter a muscle cell or adipocyte). The results of this study also suggest that the cerebral cortex and hippocampus were highly vulnerable to protein oxidation and therefore accountable to present an altered function compared to other regions like the cerebellum, medulla, and spinal cord.

The tentative conclusion thus can be drawn from the findings that oxidative stress resulting from a hyperglycemic intrauterine milieu increased the oxidative damage and alteration in the antioxidant defense during fetal and neonatal stages in the developing brain. The results of the present findings for the first time confirm that the oxidative changes that occurred during the fetal stage remain into weaning and adulthood specifically in the functional areas like the cerebral cortex and hippocampus which could in turn, bring impairments/deficits in memory and cognitive processes.

\section{ACKNOWLEDGEMENTS}

Rizwan Sharief, is grateful to Directorate of Minorities, Government of Karnataka, India, for partial financial support and awarding research fellowship

\section{AUTHORSHIP STATEMENT}

The design of the study and guidance was done by Mahaboob Basha $\mathrm{P}$ and data analysis, writing, practical aspects were carried out by Rizwan Sharief. 


\section{REFERENCES}

[1]. V. Sethna, et al., "Mother-infant interactions and regional brain volumes in infancy: an MRI study," Brain Struct Funct, vol. 222, no.5, pp. 23792388, 2017.

[2]. G. P. Kenny and R. J. Sigal , R. McGinn, "Body temperature regulation in diabetes," Temperature (Austin), vol. 3, no. 1, pp.119-145, 2016.

[3]. N. H. Cho, et al., "IDF Diabetes Atlas: Global estimates of diabetes prevalence for 2017 and projections for 2045," Diabetes Res Clin Pract, vol.138, pp. 271-281, 2018.

[4]. W. W. Hay, "Care of the infant of the diabetic mother," Current diabetes reports, vol. 12, no. 1, pp. 4-15, 2012.

[5]. B.S. Buckley, et al., "Gestational diabetes mellitus in Europe: prevalence, current screening practice and barriers to screening. A review" Diabetic medicine: a journal of the British Diabetic Association, vol.29, no. 7, pp. 844-54, 2012.

[6]. A. Fraser and D. A. Lawlor "Long-term health outcomes in offspring born to women with diabetes in pregnancy," Current diabetes reports, vol. 14, no. 5, 2014.

[7]. A. Fraser, S. M. Nelson, C. Macdonald-Wallis and D. A. Lawlor, "Associations of existing diabetes, gestational diabetes, and glycosuria with offspring IQ and educational attainment: The Avon Longitudinal Study of Parents and Children" Experimental diabetes research, 2012.

[8]. A. Ornoy, N. Ratzon, C. Greenbaum, A. Wolf and M. Dulitzky, "School-age children born to diabetic mothers and to mothers with gestational diabetes exhibit a high rate of inattention and fine and gross motor impairment," Journal of pediatric endocrinology \& metabolism, vol. 14, no. 1, pp. 681689, 2001.

[9]. Hanssen MJ, et al., "Short-term cold acclimation improves insulin sensitivity in patients with type 2 diabetes mellitus," Nat Med, vol. 21, pp. 863-865, 2015.

[10]. X. Wang et al., "Effects of Mild Chronic Intermittent Cold Exposure on Rat Organs," Int J Biol Sci, vol. 11, no. 10, pp.1171-1180, 2015.

[11]. W. G. Niehaus and B. Samuelsson, "Formation of malonaldehyde from phospholipid arachidonate during microsomal lipid peroxidation," Eur J Biochem. vol. 6, no.1, pp. 126-130, 1968.

[12]. A. Z. Reznick and L. Packer, "Oxidative damage to proteins: spectrophotometric method for carbonyl assay," Methods Enzymol, vol. 233, pp. 357-363, 1994.

[13]. M. F. Lou, L. L. Poulsen and D. M. Ziegler, "Cellular protein-mixed disulfides," Methods Enzymol, vol. 143, pp. 124-129. 1987.

[14]. P. M. Basha and S M Saumya, "Influence of Fluoride on Streptozotocin Induced Diabetic Nephrotoxicity in Mice: Protective Role of Asian Ginseng (Panax Ginseng) \& Banaba (Lagerstroemia Speciosa) on
Mitochondrial Oxidative Stress," Indian J Med Res, vol. 137, no. 2, pp. 370-9 2013.

[15].P. M. Basha and A. Poojary, "Mitochondrial Dysfunction in Aging Rat Brain Regions upon Chlorpyrifos Toxicity and Cold Stress: An Interactive Study," Cell Mol Neurobiol, vol. 34, pp.737-756, 2014.

[16]. M. Huerta-Cervantes et al., "Gestational Diabetes Triggers Oxidative Stress in Hippocampus and Cerebral Cortex and Cognitive Behavior Modifications in Rat Offspring: Age- and SexDependent Effect," Nutrients, vol. 12, 2020.

[17]. P. L. Zusterzeel, T. P. Mulder, W. H. Peters, S. A. Wiseman and E. A. Steegers, "Plasma protein carbonyls in nonpregnant, healthy pregnant and preeclamptic women." Free Radic Res, vol. 33, no.5, pp. 471-476, 2000.

[18]. E. R. Stadtman and B. S. Berlett, "Reactive oxygenmediated protein oxidation in aging and disease," Drug Metab Rev, vol. 30, no. 2, pp. 225-243, 1998.

[19]. A. Phaniendra, J. Dinesh Babu and P. Latha, "Free Radicals: Properties, Sources, Targets, and Their Implication in Various Diseases, "Indian $J$ Clin Biochem, vol. 30, no. 1, pp. 11-26, 2015.

[20]. B. S Berlett and E. R Stadtman, "Protein oxidation in aging, disease, and oxidative stress," J Biol Chem, vol. 272, no. 33, pp, 20313-6, 1997.

[21].S. Uhlig and A. Wendel, "The physiological consequences of glutathione variations," Life Sci, vol. 51, pp. 1083-94, 1992.

[22]. J. S. Stamler, A. Slivka, "Biological chemistry of thiols in the vasculature and in vascular-related disease," Nutr Rev, vol. 54, no. 1, pp. 1-30. 1996;

[23]. J. Navarro et al., "Blood glutathione as an index of radiation-induced oxidative stress in mice and humans," Free Radic Biol Med, vol. 22, no. 7, pp.1203-1209, 1997.

[24]. P. Petrone, J. A. Asensio and C. P. Marini, "Management of accidental hypothermia and cold injury," Curr Probl Surg, vol. 51, no. 10, pp. 417-31, 2014.

[25]. A. Fenzl and F. W. Kiefer, "Brown adipose tissue and thermogenesis," Horm Mol Biol Clin Investig, vol.19, no 1, pp. 25-37, 2014.

[26]. A. L Poher, J. Altirriba, C. Veyrat-Durebex, F. Rohner-Jeanrenaud, "Brown adipose tissue activity as a target for the treatment of obesity/insulin resistance," Front Physiol, vol. 6, pp.1-10, 2015.

[27]. P. Nuutila, "Brown adipose tissue thermogenesis in humans," Diabetologia, vol. 56, no. 10, pp. 2110 2112, 2013. 